\title{
Investigation of the anti-asthmatic activity of Oridonin on a mouse model of asthma
}

\author{
JING WANG $^{1}$, FENGSEN LI ${ }^{1}$, JIANBING DING ${ }^{1,2}$, GE TIAN $^{1}$, MIN JIANG $^{1}$, ZHEN GAO $^{1}$ and EHBAL TUYGHUN ${ }^{1}$ \\ ${ }^{1}$ Xinjiang Key Laboratory of Respiratory Disease Research, TCM Affiliated Hospital of Xinjiang Medical University; \\ ${ }^{2}$ College of Basic Medicine, Xinjiang Medical University, Ürümqi, Xinjiang 830011, P.R. China
}

Received May 27, 2015; Accepted June 14, 2016

DOI: $10.3892 / \mathrm{mmr} .2016 .5485$

\begin{abstract}
Oridonin is an extract obtained from a traditional Chinese medicinal herb, Xihuangcao. Previous studies have demonstrated that Oridonin exerts various pharmaceutical effects, such as anti-tumor and immunosuppressive effects, as well as modulating cytokine balance. The present study identified that Oridonin could regulate the $T_{h} 1 / T_{h} 2$ cytokine balance in mice. However, as the anti-asthmatic effect of Oridonin is currently unknown a mouse model of asthma was used in the present study. BALB/c mice were sensitized using ovalbumin (OVA), then the sensitized mice were treated with Oridonin prior to OVA challenge. The in vivo study indicated that Oridonin decreased the OVA-induced airway hyper-responsiveness significantly $(\mathrm{P}<0.05)$. In addition, the results indicated that in Oridonin-treated mice, the eosinophil number and total inflammatory cell number in bronchoalveolar lavage (BAL) fluid decreased significantly in the Oridonin group when compared with the control group. Further study indicated that Oridonin significantly decreased the level of inflammatory cytokines, which were induced by OVA, in BAL fluid. Histological studies were performed to evaluate the effect of Oridonin on eosinophilia and mucus in the airway, the results indicated that Oridonin significantly inhibited the eosinophilia and mucus production in the lungs. Therefore the present study demonstrated that Oridonin regulates $T_{h} 1 / T_{h} 2$ balance in mice and exhibited anti-asthmatic effects in a mouse model of asthma. These findings indicate that Oridonin
\end{abstract}

Correspondence to: Dr Fengsen Li, Xinjiang Key Laboratory of Respiratory Disease Research, TCM Affiliated Hospital of Xinjiang Medical University, 116 Huang He Road, Wulumuqi, Ürümqi, Xinjiang 830011, P.R. China

E-mail: fengsenl@126.com

Dr Jianbing Ding, College of Basic Medicine, Xinjiang Medical University, 116 Huang He Road, Wulumqi, Ürümqi, Xinjiang 830011, P.R. China

E-mail: jianbingd@126.com

Key words: Oridonin, anti-asthmatic, ovalbumin, asthma, mouse model, cytokine may serve as a potential therapeutic compound for the treatment of asthma in future.

\section{Introduction}

Asthma is a chronic airway inflammatory disease, which is associated with various cell and cellular components. The majority of asthma occurs in the developing world and it often begins in childhood. The rates of asthma have markedly increased since the $1960 \mathrm{~s}$, in 2013 there were 242 million asthma patients, and it resulted in $\sim 489,000$ deaths $(1,2)$. It is characterized by inflammation, eosinophilia and mucus hypersecretion in the airways and lungs. Goblet cells may contribute to the immunoglobulin E secretion and airway hyperresponsiveness (AHR) (3). It is reported that eosinophils, mast cells and lymphocytes also contribute to asthma (4-7). Previous studies indicated that cytokines, such as interleukin (IL)-4, IL-5 and IL-13 produced by type $2 \mathrm{~T}$ helper $\left(\mathrm{T}_{\mathrm{h}}\right)$ cells could regulate eosinophilia in the airways (8-10). Conversely, eosinophils may produce cytokines and chemokines, which could contribute to the development of asthma $(11,12)$. Therefore, cytokines and other mediators are considered to be particularly important in the treatment of asthma, and regulation of cytokines and chemokines may contribute to the treatment of asthma (13-16). Furthermore, cytokine and chemokine regulation may improve the efficacy of clinical treatment methods $(17,18)$.

Oridonin (Fig. 1), an extract from the traditional Chinese medicinal herb, Xihuangcao is widely used in China (19). It has been shown to exert various pharmaceutical effects, such as anti-tumor (20), anti-proliferation (21), anti-inflammatory and antiviral effects. In addition, Oridonin may be administered for the treatment of upper respiratory tract infection (22). In China Oridonin has been administered for the treatment of inflammatory diseases for hundreds of years and has become one of the most popular herbs used clinically (23). Recent studies have indicated that Oridonin exhibits significant immunosuppressive effects in mice and modulates the $\mathrm{T}_{\mathrm{h}} 1 / \mathrm{T}_{\mathrm{h}} 2$ balance in in vitro studies with rats $(24,25)$. However, whether Oridonin could be used in the treatment of asthma, and the underlying mechanisms of its anti-inflammatory activity in mice and rats remain unknown.

In the present study, an ovalbumin (OVA)-induced asthma mouse model was used to evaluate the anti-asthmatic effect of 
Oridonin and to elucidate the mechanism of its anti-inflammatory effect. In addition, the in vitro effects of Oridonin on $\mathrm{T}_{\mathrm{h}} 1 / \mathrm{T}_{\mathrm{h}} 2$ cytokine balance in mice were assessed.

\section{Materials and methods}

Oridonin. Oridonin (purity, >98.0\%; Fig. 1) was purchased from Zelang Medical Technology Co.Ltd. (Nanjing, China). Oridonin was dissolved in dimethyl sulfoxide (DMSO; Sigma-Aldrich, St. Louis, MO, USA). In the cell assay-based studies, the final DMSO concentration was $<0.01 \%$.

Animals. A total of 32 male BALB/c mice (age, 4-6 weeks) were purchased from Shanghai SLAC Laboratory Animal Co., Ltd. (Shanghai, China). The mice were maintained under specific pathogen-free conditions for at least 7 days prior to the ex vivo and in vivo studies. The mice had access to food and water ad libitum, and were maintained at $20-26^{\circ} \mathrm{C}$ under a $12-\mathrm{h}$ light/dark cycle. The mice were divided into 4 groups of 8 , as follows: i) phosphate-buffered saline (PBS; Invitrogen; Thermo Fisher Scientific, Inc.) group; ii) OVA group; iii) Oridonin-L; and iv) Oridonin-H. All animal studies were performed according to the Guide for the Care and Use of Laboratory Animals (26).

Lymphocyte preparation. The BALB/c mice were sacrificed with carbon dioxide and their spleens were harvested. The spleens were sliced into small pieces and pulverized using a syringe. The cell suspension in RPMI 1640 medium (Invitrogen; Thermo Fisher Scientific, Inc., Waltham, MA, USA) was filtered through a $70-\mu \mathrm{m}$ cell strainer (BD Biosciences, Franklin Lakes, NJ, USA). Red blood cells were lysed using 10 mM EDTA (Invitrogen; Thermo Fisher Scientific, Inc.), as previously described (27). The cells were then washed with PBS and re-suspended in PBS. NycoPrep ${ }^{\text {TM }}$ 1.077A (Axis-Shield PoC, Oslo, Norway) was used to isolate the lymphocytes from the spleen cells as previously described (25). Finally, the lymphocytes were cultured in RPMI-1640 medium supplemented with $10 \%$ fetal bovine serum. The primary mouse spleen cells were stimulated with $5 \mu \mathrm{g} / \mathrm{ml}$ concanavalin A (Sigma-Aldrich) prior to Oridonin treatment.

Enzyme-linked immunosorbent assay (ELISA). Supernatant and bronchoalveolar lavage (BAL) fluid from the different groups was harvested by flushing the lung with $0.5 \mathrm{ml}$ ice-cold PBS, following sacrifice with carbon dioxide, as described below and stored at $-80^{\circ} \mathrm{C}$. Cytokine and chemokine levels in the cell supernatant or BAL fluid were measured by ELISA assay. The ELISA kits were purchased from R\&D Systems, Inc. (Minneapolis, MN, USA).

Mouse asthma model. On day 0 and 14, mice (8 mice/group) were injected with $20 \mu \mathrm{g}$ OVA (Sigma-Aldrich). On day 28, 29 and 30, the mice were administrated with Oridonin (10 or $20 \mathrm{mg} / \mathrm{kg}$ ) or vehicle $(0.5 \%$ sodium salt of carboxy methyl cellulose), $1 \mathrm{~h}$ after administration the mice were challenged with OVA (1\%). The administration doses were established according to a previous study (24).

Measurement of AHR. AHR was evaluated in the Oridonin-treated or vehicle group mice by whole-body

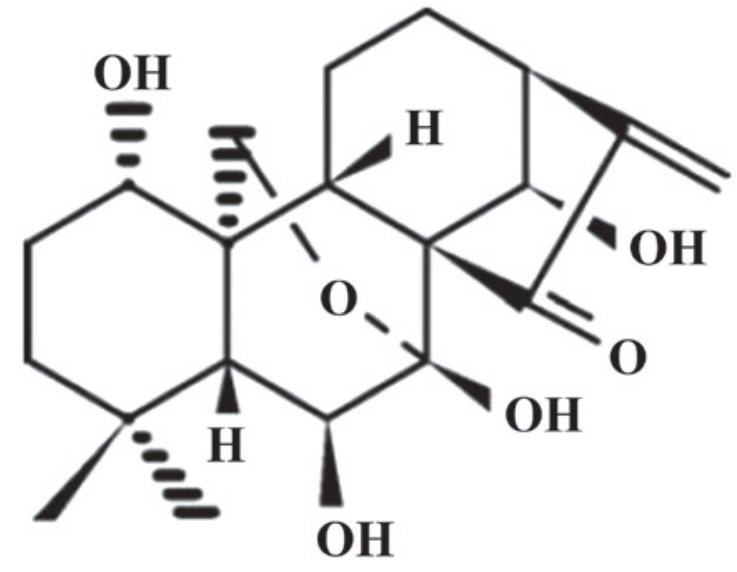

Figure 1. Chemical structure of Oridonin.

plethysmography $24 \mathrm{~h}$ after the final OVA challenge. The mice were placed in separate chambers and exposed to 20,40,60 and $80 \mathrm{mg} / \mathrm{ml}$ aerosolized methacholine solution (Sigma-Aldrich). The bronchoconstriction was then measured for $5 \mathrm{~min}$. The highest Penh value obtained in each group was expressed as a basal Penh value compared with the PBS challenge, which served as the control.

$B A L$. Twenty-four-hours subsequent to the final aerosol OVA challenge, the BALB/c mice were sacrificed with carbon dioxide and the trachea of the mice were incised. Ice-cold PBS was injected into the lungs of the mice through the trachea and the BAL fluid was subsequently harvested. Each mouse was injected with PBS three times. Cytospin slides (Thermo Fisher Scientific, Inc.) were stained in a modified Wright stain (Sigma-Aldrich); the number of inflammatory corpuscles and total cells were counted using a hemocytometer (Countess II FL Automated Cell Counter; Thermo Fisher Scientific, Inc.).

Histologic examination. All the mice were sacrificed with carbon dioxide and the lungs were harvested. The lungs were infused, via the trachea, with formalin (Sigma-Aldrich) overnight. The tissues were then paraffinized (Sigma-Aldrich) and sliced into 7- $\mu \mathrm{m}$ sections. Hematoxylin and eosin (H\&E; Beyotime Institute of Biotechnology) staining was performed to evaluate eosinophil infiltration and periodic acid-Schiff staining was performed to measure mucus production. Quantitative analysis of eosinophil infiltration and mucus production was performed as previously described (28). The scoring system for cell infiltration was: 0 , no cells; 1 , few cells; 2 , a ring of cells that were one cell layer deep; 3 , a ring of cells 2-4 cell layers deep; 4 , a ring of cells $>4$ cells deep. Goblet cell hyperplasia in the airway epithelium was quantified based on a five-point system: 0 , no goblet cells; $1,<25 \%$ of the epithelium; $2,25-50 \%$ of the epithelium; $3,50-75 \%$ of the epithelium; 4 , $>75 \%$ of the epithelium.

Statistical analysis. Data were presented as means \pm standard deviation for the in vitro experiments and means \pm standard error of the mean for in vivo study. Statistical analysis was performed with the Student's t-test using SPSS 14.0 (SPSS, Inc., Chicago, IL, USA) to determine the differences between 

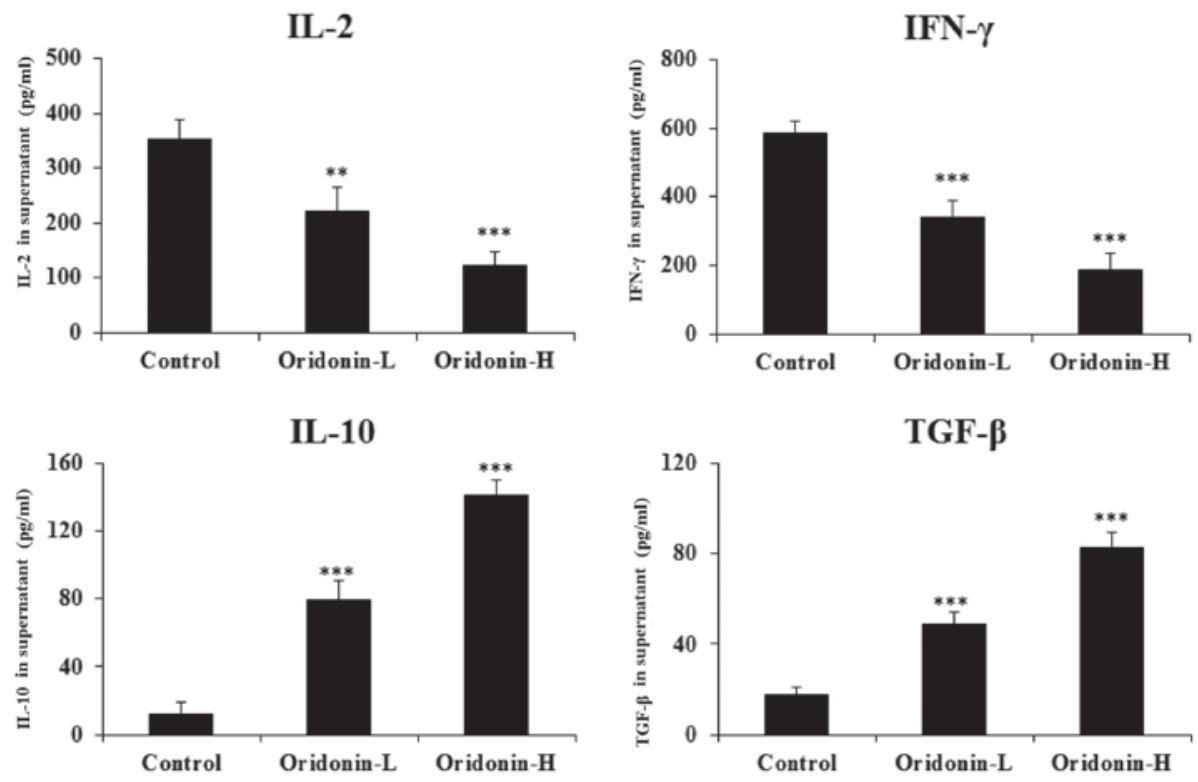

Figure 2. Effect of Oridonin on $T_{h} 1 / T_{h} 2$ cytokines in concanavalin A-stimulated mouse spleen cells from one mouse. Expression levels of $T_{h} 1$ and $T_{h} 2$ cytokines in the supernatant of different groups [PBS control; Oridonin-L, asthmatic mice treated with Oridonin (10 mg/kg); Oridonin- $\mathrm{H}$, asthmatic mice treated with Oridonin $(20 \mathrm{mg} / \mathrm{kg})]$ were measured by enzyme-linked immunosorbent assay. All tests were performed in triplicate and the data are presented as means \pm standard deviation. ${ }^{* *} \mathrm{P}<0.01$ and ${ }^{* * *} \mathrm{P}<0.001$ vs. control. IL, interleukin; IFN, interferon; TGF, transforming growth factor.

groups. $\mathrm{P}<0.05$ was considered to indicate a statistically significant difference.

\section{Results}

Oridonin treatment modulated the $T_{h} 1 / T_{h} 2$ balance in mice. A previous study indicated that Oridonin regulated the $T_{h} 1 / T_{h} 2$ balance in rats (25). The present study aimed to investigate whether Oridonin modulates the $T_{h} 1 / T_{h} 2$ cytokine balance in mice. Our preliminary experiment indicated that Oridonin dose-dependently decreased the viability of the primary mice spleen cells, and that the half maximal inhibitory concentration is $\sim 37 \mathrm{mM}$ (data not shown). In the present study primary mice spleen cells were cultured, stimulated with concanavalin A and treated with Oridonin $(12.5$ or $25 \mathrm{mM})$. The supernatant was then harvested for ELISA. The results indicated that Oridonin treatment significantly decreased the $T_{h} 1$ cytokine level [interferon (IFN)- $\gamma$ and IL-2; $\mathrm{P}<0.001]$ in a dose-dependent manner, and significantly increased the $T_{h} 2$ cytokine level (transforming growth factor; TGF- $\beta$ and IL-10; $\mathrm{P}<0.001$ ), also in a dose-dependent manner, in the mice spleen cell culture supernatant (Fig. 2). Thus, the results indicate that Oridonin regulates the $T_{h} 1 / T_{h} 2$ cytokine balance in mice.

Effects of Oridonin on AHR. Previous studies have indicated that asthma is associated with the $\mathrm{T}_{\mathrm{h}} 1 / \mathrm{T}_{\mathrm{h}} 2$ balance (29), and that Oridonin may modulate the rat $\mathrm{T}_{\mathrm{h}} 1 / \mathrm{T}_{\mathrm{h}} 2$ balance in vitro (25). The present study demonstrated that Oridonin modulated the mice $\mathrm{T}_{\mathrm{h}} 1 / \mathrm{T}_{\mathrm{h}} 2$ balance in vitro, therefore, the effect of Oridonin was investigated in a mouse model of asthma. Asthma is defined as excessive narrowing of the airways when challenged with contractile agonists; OVA is a contractile agonist. To investigate the anti-asthmatic effect of Oridonin on asthma in mice, a mouse model of asthma was established with



Figure 3. Effect of Oridonin on AHR to inhaled methacholine. AHR was measured by whole-body plethysmography. The groups were as follows: PBS, PBS-challenged mice; OVA, OVA-challenged mice; Oridonin-L, asthmatic mice treated with Oridonin $(10 \mathrm{mg} / \mathrm{kg})$; Oridonin- $\mathrm{H}$, asthmatic mice treated with Oridonin $(20 \mathrm{mg} / \mathrm{kg})$. Values are expressed as the mean \pm standard error of the mean. " $\mathrm{P}<0.05$ vs. OVA group; $\mathrm{n}=8$. PBS, phosphate-buffered saline; OVA, ovalbumin; AHR, airway hyperresponsiveness.

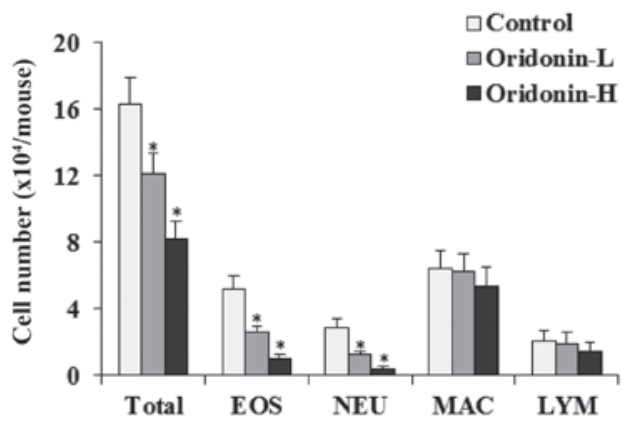

Figure 4. Effects of Oridonin on BAL fluid cell infiltration. BAL fluids were collected $24 \mathrm{~h}$ after the final OVA challenge. Differential cell count was performed on a minimum of 500 cells to identify EOS, NEU, MAC, and LYM. Control, OVA-challenged mice; Oridonin-L, asthmatic mice treated with Oridonin $(10 \mathrm{mg} / \mathrm{kg})$; Oridonin- $\mathrm{H}$, asthmatic mice treated with Oridonin $(20 \mathrm{mg} / \mathrm{kg})$. Values are expressed as the mean \pm standard error of the mean. ${ }^{*} \mathrm{P}<0.05$ vs. the control group. BAL, bronchoalveolar lavage; EOS, eosinophil; NEU, neutrophil; MAC, macrophage; LYM, lymphocyte; OVA, ovalbumin. 

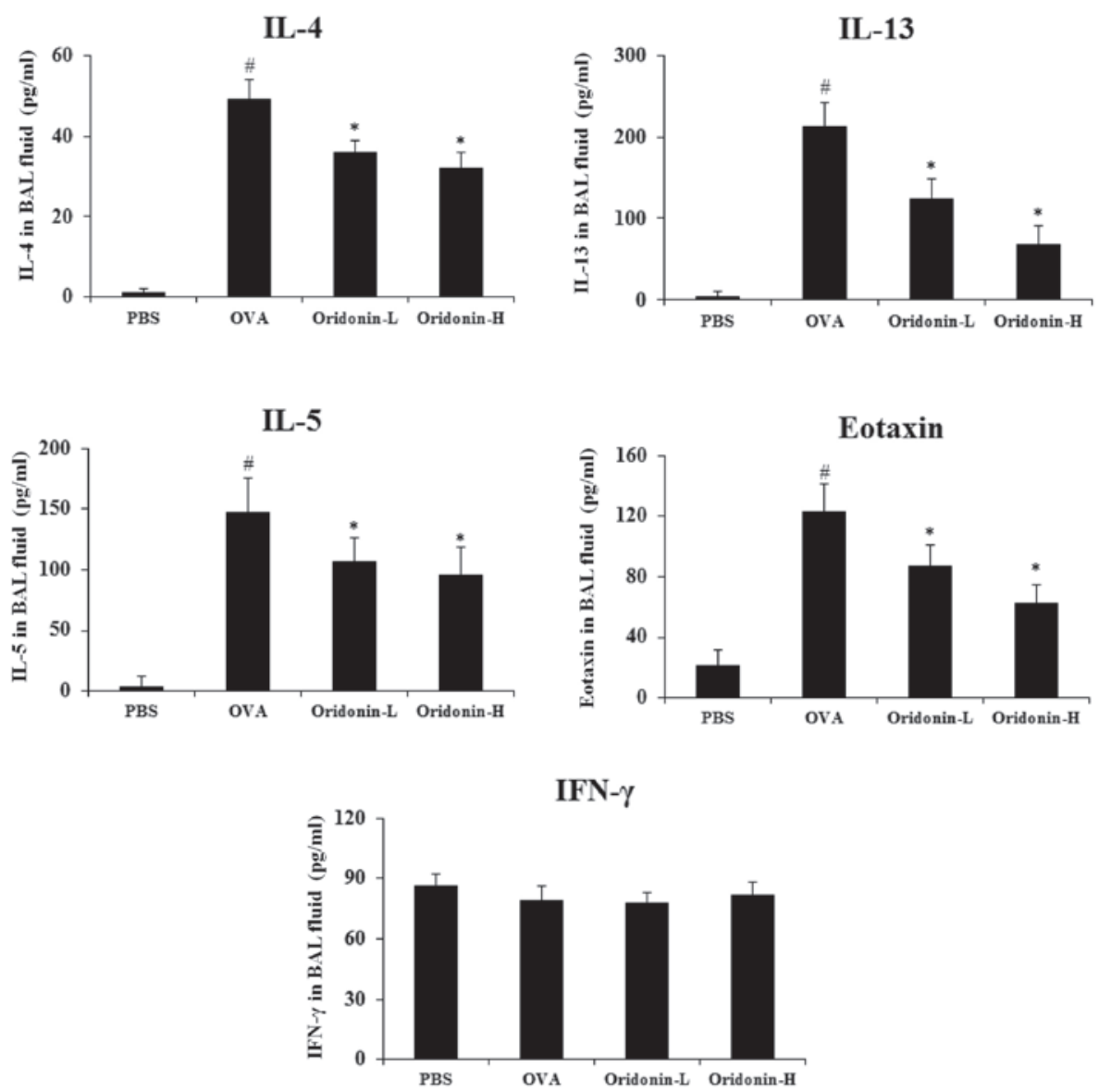

Figure 5. Effects of Oridonin on cytokine and chemokine levels in BAL fluid. BAL fluids were collected $2 \mathrm{~h}$ after the final OVA challenge. Levels of IL-4, IL-5, IL-13, eotaxin and IFN- $\gamma$ were analyzed using enzyme-linked immunosorbent assay. PBS, PBS-challenged mice; OVA, OVA-challenged mice; Oridonin-L, asthmatic mice treated with Oridonin $(10 \mathrm{mg} / \mathrm{kg})$; Oridonin- $\mathrm{H}$, asthmatic mice treated with Oridonin $(20 \mathrm{mg} / \mathrm{kg})$. Values are expressed as the mean \pm standard error of the mean. "P<0.05 vs. PBS group; "P<0.05 vs. OVA group. IL, interleukin; IFN, interferon; PBS, phosphate-buffered saline; OVA, ovalbumin; BAL, bronchoalveolar lavage.

methacholine to induce the AHR in OVA-challenged mice, and AHR was measured. Mice that had been sensitized with OVA continuously for three days were stimulated with methacholine to induce AHR. The control group was stimulated with PBS. The results indicate that Oridonin significantly inhibited methacholine-induced AHR when compared with the vehicle group ( $\mathrm{P}<0.05$; Fig. 3$)$.

Effects of Oridonin on eosinophil recruitment. Eosinophil recruitment is an important characteristic in asthma (30). When challenged with OVA, numerous leukocytes were recruited in the lung (31), therefore, the effect of Oridonin on eosinophil recruitment was investigated in BAL fluid of OVA-challenged mice. The OVA-challenged mice lungs were flushed with cold PBS $48 \mathrm{~h}$ after the last challenge and cells in the BAL fluid were counted. The results indicated that Oridonin significantly decrease the number of eosinophils, neutrophils and total cells in BAL fluid when compared with the control group $(\mathrm{P}<0.05)$. The effects of Oridonin on the number of macrophages and lymphocytes were marginal (Fig. 4).

Effect of Oridonin on cytokine levels in BAL fluid. Humoral immune response usually causes the allergic reaction, when exposed to an allergen the immune cells secrete a series of cytokines and these cytokines, such as IL-4 and IL-13 contribute to the allergic condition (32). Kim et al (33) indicated that the imbalance of IL-5, eotaxin and IFN- $\gamma$ was associated with asthma. Therefore, the present study analyzed the effect of Oridonin on cytokine levels in the BAL fluid of mice. The lungs of OVA-challenged mice were lavaged with cold PBS $48 \mathrm{~h}$ after the final challenge and the BAL fluid was collected. The ELISA assay was performed to evaluate the level of the associated cytokines in the mice BAL fluid according to the manufacturer's instructions. The findings demonstrated that the level of IL-4, IL-13, IL-5 and eotaxin increased significantly in the BAL fluid of the OVA-challenged mice $(\mathrm{P}<0.05)$. The results indicated that Oridonin treatment significantly inhibited the IL-4, IL-13, IL-5 and eotaxin levels in BAL fluid, but exerted a marginal effect on IFN- $\gamma$ (Fig. 5). These results indicate that Oridonin modulated the level of cytokines produced by immune cells in asthmatic mice.

Effects of Oridonin on eosinophil infiltration and mucus production. Significant migration of inflammatory cells (eosinophils) to peribronchiolar tissues was observed in the mice that had been challenged with OVA (31). Therefore, the effect of Oridonin on the eosinophil infiltration and mucus production was investigated in the lungs of asthmatic mice. Twenty-four hours after the last OVA challenge, mice were sacrificed and their lungs were harvested. Lung tissues were 
A
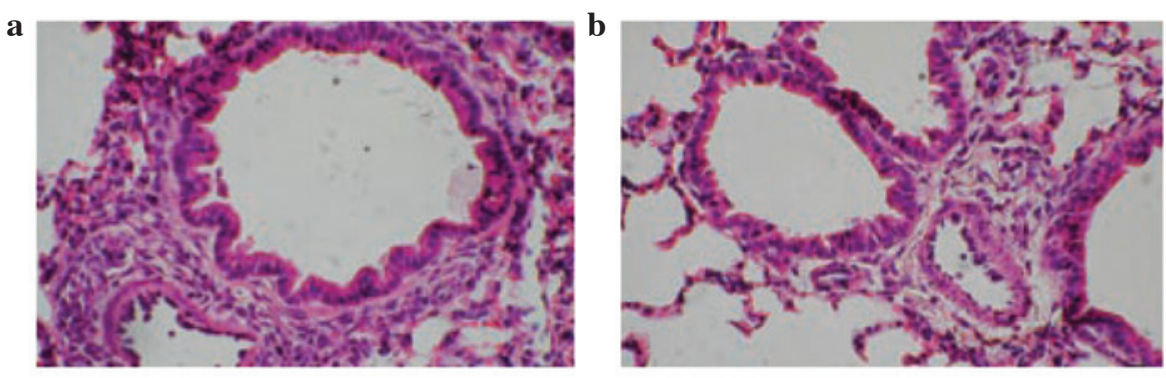

c

d
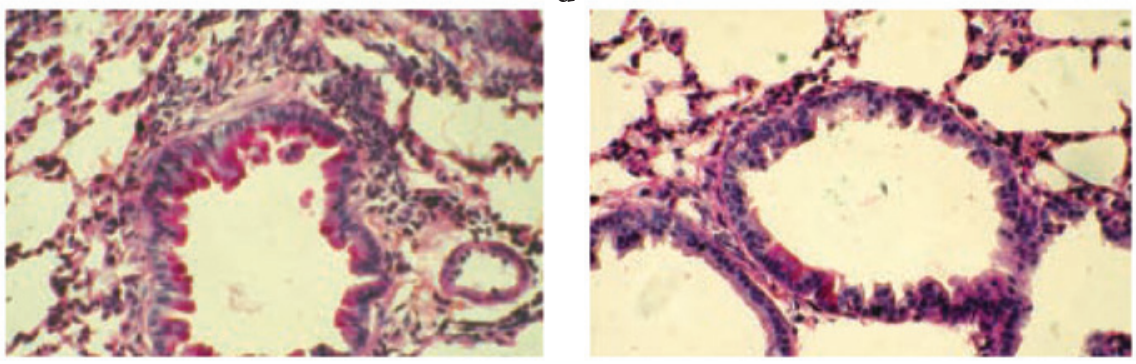

$\mathbf{B}$

Inflammation score

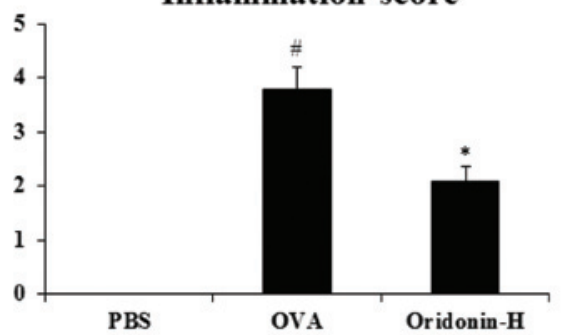

C

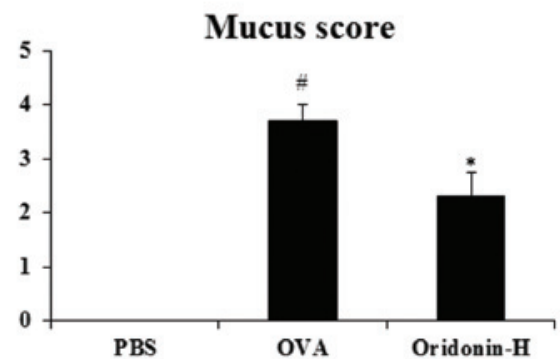

Figure 6. Effects of Oridonin on lung tissue eosinophilia and mucus production. (A) Histological examination of (a) OVA-challenged and (b) Oridonin-H mice lung tissue eosinophilia and (c) OVA-challenged and (d) Oridonin-H mice mucus secretion demonstrated that administration of Oridonin significantly decreased eosinophilia and mucus secretion in lung tissue. Magnification, x400. Quantitative analyses of (B) inflammatory cell infiltration and (C) mucus production in lung sections. Lung tissue samples were fixed, sectioned at 7- $\mu \mathrm{m}$ thickness and stained with hematoxylin and eosin, for tissue eosinphilia or periodic acid-Schiff, for mucus production. Oridonin- $\mathrm{H}$, asthmatic mice treated with Oridonin $(20 \mathrm{mg} / \mathrm{kg})$. Values are expressed as the mean \pm standard error of the mean. ${ }^{\#} \mathrm{P}<0.05$ vs. PBS group; ${ }^{*} \mathrm{P}<0.05$ vs. OVA group. OVA, ovalbumin; PBS, phosphate-buffered saline.

stored in formalin and histologically examined by a pathologist (Fig. 6A). The histopathological examination results indicated that following challenge with OVA, the eosinophil infiltration and mucus production were significantly increased compared to the PBS group $(\mathrm{P}<0.05)$. However, following administration of oridonin the inflammation score decreased from 3.8 to $2.1(\mathrm{P}<0.05)$ and the mucus score decreased from 3.7 to 2.3 $(\mathrm{P}<0.05)$. These results indicate that Oridonin significantly inhibited eosinophil infiltration (Fig. 6B) and mucus production (Fig. 6C) in the asthmatic lung when compared with that of the control group.

\section{Discussion}

Asthma is a chronic disease of the airways. It is characterized by airway inflammation, the increased secretion of mucus and AHR (34). Previous studies have indicated that immune cells (T, B, and mast cells and eosinophils), cytokines and chemokines contribute to inflammatory responses $(34,35)$. $\mathrm{T}$ helper cell type 2 produces $\mathrm{T}_{\mathrm{h}} 2$ cytokines, which are associated with the maturation of $\mathrm{B}$ cells. $\mathrm{T}_{\mathrm{h}} 2$ cytokines include IL-4, IL-5, and IL-13 and these cytokines are important in humoral immune responses. Furthermore, chemokines are essential for eosinophil infiltration into lung tissues.
Therefore modulation of cytokine and chemokine balance may be a potential therapeutic strategy for asthma.

Oridonin is a chemical compound extracted from the traditional Chinese medicinal herb, Xihuangcao, which is a popular herbal medicine in China (19). Previous studies have indicated that Oridonin may cure lymphoid malignancies (36), leukemia (37) and auto immune disease (24). However, to the best of our knowledge, the effects of Oridonin on asthma have not been reported.

A previous study indicated that Oridonin regulated the $\mathrm{T}_{\mathrm{h}} 1 / \mathrm{T}_{\mathrm{h}} 2$ balance in rats (25); in the present study, it was demonstrated that Oridonin also modulated $\mathrm{T}_{\mathrm{h}} 1 / \mathrm{T}_{\mathrm{h}} 2$ balance in mice. Therefore, Oridonin was evaluated in vivo in a mouse asthma model to assess the anti-asthmatic effects. Our acute toxicity study indicated that when administrated with $50 \mathrm{mg} / \mathrm{kg}$ Oridonin, the mice survived and no loss of body weight was observed (data not shown). The results indicated that Oridonin significantly decreased the AHR in asthmatic mice and decreased the eosinophil, neutrophil and total cell number in BAL fluid. To further investigate the potential mechanisms of action of Oridonin, cytokine and chemokine production was assessed in the BAL fluid of mice.

The levels of $\mathrm{T}_{\mathrm{h}} 2$ cytokines and chemokines increased in the OVA-challenged mice. IL-5 is important in the survival 
and recruitment of eosinophils; while IL-13 is significant in AHR, eosinophilic infiltration and mucus secretion $(35,38)$. In addition, chemokines contribute to eosinophil migration in the airways, with Regulated on Activation, Normal T Cell Expressed and Secreted and eotaxin as the two most important chemokines. Adhesion molecules are also involved in eosinophil migration (39). These factors, $\mathrm{T}_{\mathrm{h}} 2$ cytokines, chemokines and adhesion molecules, may contribute to AHR in asthma together (40). The present study indicates that Oridonin significantly inhibited the IL-4, IL-13, IL-5 and eotaxin levels in BAL fluid. These results indicate that the regulation of cytokine balance may contribute to asthma in mice. However, the underlying mechanisms remain unclear and further investigation is required.

In conclusion, the present study demonstrates that Oridonin regulated the cytokine balance in OVA-challenged mice, inhibited AHR and reduced lung eosinophilia, mucus hypersecretion in asthmatic mice. These findings suggest that Oridonin may serve as a potential novel compound for the treatment of asthma.

\section{Acknowledgements}

The present study was supported by the Innovation and Development Fund for Scientific Research Institution of Xinjiang Uyghur Autonomous Region: The Construction of Traditional Chinese Medicine Base for Transformation and Prevention of Respiratory System Disease in Xinjiang (grant. no. 2015008).

\section{References}

1. Global Burden of Disease Study 2013 Collaborators: Global, regional, and national incidence, prevalence, and years lived with disability for 301 acute and chronic diseases and injuries in 188 countries, 1990-2013: A systematic analysis for the Global Burden of Disease Study 2013. Lancet 386: 743-800, 2015.

2. GBD 2013 Mortality and Causes of Death Collaborators: Global, regional, and national age-sex specific all-cause and cause-specific mortality for 240 causes of death, 1990-2013: a systematic analysis for the Global Burden of Disease Study 2013 Lancet 385: 117-171, 2015.

3. Elias JA, Lee CG, Zheng T, Ma B, Homer RJ and Zhu Z: New insights into the pathogenesis of asthma. J Clin Invest 111: 291-297, 2003.

4. Robinson DS, Hamid Q, Ying S, Tsicopoulos A, Barkans J, Bentley AM, Corrigan C, Durham SR and Kay AB: Predominant TH2-like bronchoalveolar T-lymphocyte population in atopic asthma. N Engl J Med 326: 298-304, 1992.

5. Okayama Y, Ra C and Saito H: Role of mast cells in airway remodeling. Curr Opin Immunol 19: 687-693, 2007.

6. Holgate ST: Epithelium dysfunction in asthma. J Allergy Clin Immunol 120: 1233-1244; quiz 1245-1246, 2007.

7. McFadden ER Jr: Acute severe asthma. Am J Respir Crit Care Med 168: 740-759, 2003.

8. Sinigaglia F and D'Ambrosio D: Regulation of helper T cell differentiation and recruitment in airway inflammation. Am J Respir Crit Care Med 162: S157-S160, 2000.

9. Broide DH: Immunologic and inflammatory mechanisms that drive asthma progression to remodeling. J Allergy Clin Immunol 121: 560-570; quiz 571-572, 2008.

10. Cho JY, Miller M, Baek KJ, Han JW, Nayar J, Lee SY, McElwain K, McElwain S, Friedman S and Broide DH: Inhibition of airway remodeling in IL-5-deficient mice. J Clin Invest 113: 551-560, 2004

11. Pascual RM and Peters SP: Airway remodeling contributes to the progressive loss of lung function in asthma: An overview. J Allergy Clin Immunol 116: 477-486; quiz 487, 2005.
12. Flood-Page P, Menzies-Gow A, Phipps S, Ying S, Wangoo A, Ludwig MS, Barnes N, Robinson D and Kay AB: Anti-IL-5 treatment reduces deposition of ECM proteins in the bronchial subepithelial basement membrane of mild atopic asthmatics. J Clin Invest 112: 1029-1036.

13. Panettieri RA Jr: Cellular and molecular mechanisms regulating airway smooth muscle proliferation and cell adhesion molecule expression. Am J Respir Crit Care Med 158: S133-S140, 1998.

14. Jeffery PK: Remodeling in asthma and chronic obstructive lung disease. Am J Respir Crit Care Med 164: S28-S38, 2001.

15. Wenzel S: Severe asthma in adults. Am J Respir Crit Care Med 172: 149-160, 2005.

16. Tagaya $E$ and Tamaoki J: Mechanisms of airway remodeling in asthma. Allergol Int 56: 331-340, 2007.

17. Mauad T, Bel EH and Sterk PJ: Asthma therapy and airway remodeling. J Allergy Clin Immunol 120: 997-1009; quiz 1010-1011, 2007.

18. Vanacker NJ, Palmans E, Kips JC and Pauwels RA: Fluticasone inhibits but does not reverse allergen-induced structural airway changes. Am J Respir Crit Care Med 163: 674-679, 2001.

19. Liu JJ, Huang RW, Lin DJ, Wu XY, Peng J, Pan XL, Song YQ, Lin Q, Hou M, Wang DN, et al: Oridonin-induced apoptosis in leukemia K562 cells and its mechanism. Neoplasma 52: 225-230, 2005.

20. Chen S, Gao J, Halicka HD, Huang X, Traganos F and Darzynkiewicz Z: The cytostatic and cytotoxic effects of oridonin (Rubescenin), a diterpenoid from Rabdosia rubescens, on tumor cells of different lineage. Int J Oncol 26: 579-588, 2005.

21. Ren KK, Wang HZ, Xie LP, Chen DW, Liu X, Sun J, Nie YC and Zhang RQ: The effects of oridonin on cell growth, cell cycle, cell migration and differentiation in melanoma cells. J Ethnopharmacol 103: 176-180, 2006.

22. Liu JJ, Wu XY, Peng J, Pan XL and Lu HL: Antiproliferation effects of oridonin on HL-60 cells. Ann Hematol 83: 691-695, 2004.

23. Xu Y,Xue Y,Wang Y,Feng D, Lin S and Xu L: Multiple-modulation effects of Oridonin on the production of proinflammatory cytokines and neurotrophic factors in LPS-activated microglia. Int Immunopharmacol 9: 360-365, 2009.

24. Liu J, Yang F, Zhang Y and Li J: Studies on the cell-immunosuppressive mechanism of Oridonin from Isodon serra. Int Immunopharmacol 7: 945-954, 2007.

25. Hu AP, Du JM, Li JY and Liu JW: Oridonin promotes CD4+/CD25+ Treg differentiation, modulates Th1/Th2 balance and induces HO-1 in rat splenic lymphocytes. Inflamm Res 57: $163-170,2008$.

26. Committee for the Update of the Guide for the Care and Use of Laboratory Animals: Guide for the Care and Use of Laboratory Animals. 8th edition. National Academies Press, Washington, D.C., 2011.

27. Xue H, Guo H, Li YC and Hao ZM: Heme oxygenase-1 induction by hemin protects liver cells from ischemia/reperfusion injury in cirrhotic rats. World J Gastroenterol 13: 5384-5390, 2007.

28. Myou S, Leff AR, Myo S, Boetticher E, Tong J, Meliton AY, Liu J, Munoz NM and Zhu X: Blockade of inflammation and airway hyperresponsiveness in immune-sensitized mice by dominant-negative phosphoinositide 3-kinase-TAT. J Exp Med 198: 1573-1582, 2003.

29. Hansbro PM, Kaiko GE and Foster PS: Cytokine/anti-cytokine therapy-novel treatments for asthma? Br J Pharmacol 163: 81-95, 2011.

30. Possa SS, Leick EA, Prado CM, Martins MA and Tibério IFLC: Eosinophilic inflammation in allergic asthma. Front Pharmacol 4: 46, 2013.

31. Duan W, Chan JH, Wong CH, Leung BP and Wong WS: Anti-inflammatory effects of mitogen-activated protein kinase kinase inhibitor U0126 in an asthma mouse model. J Immunol 172: 7053-7059, 2004.

32. Abbas AK, Murphy KM and Sher A: Functional diversity of helper T lymphocytes. Nature 383: 787-793, 1996.

33. Kim CK, Kita H, Callaway Z, Kim HB, Choi J, Fujisawa T, Shin BM and Koh YY: The roles of a Th2 cytokine and CC chemokine in children with stable asthma: Potential implication in eosinophil degranulation. Pediatr Allergy Immunol 21: e697-e704, 2010.

34. Busse WW and Lemanske RF Jr: Asthma. N Engl J Med 344: 350-362, 2001.

35. Herrick CA and Bottomly K: To respond or not to respond: $\mathrm{T}$ cells in allergic asthma. Nat Rev Immunol 3: 405-412, 2003. 
36. Ikezoe T, Yang Y, Bandobashi K, Saito T, Takemoto S, Machida H, Togitani K, Koeffler HP and Taguchi H: Oridonin, a diterpenoid purified from Rabdosia rubescens, inhibits the proliferation of cells from lymphoid malignancies in association with blockade of the NF-kappaB signal pathways. Mol Cancer Ther 4: 578-586, 2005.

37. Zhou GB, Kang H, Wang L, Gao L, Liu P, Xie J, Zhang FX, Weng XQ, Shen ZX, Chen J, et al: Oridonin, a diterpenoid extracted from medicinal herbs, targets AML1-ETO fusion protein and shows potent antitumor activity with low adverse effects on $\mathrm{t}(8 ; 21)$ leukemia in vitro and in vivo. Blood 109 3441-3450, 2007.
38. Wynn TA: IL-13 effector functions. Annu Rev Immunol 21: 425-456, 2003

39. Lukacs NW: Role of chemokines in the pathogenesis of asthma. Nat Rev Immunol 1: 108-116, 2001.

40. Wills-Karp M: Immunologic basis of antigen-induced airway hyperresponsiveness. Annu Rev Immunol 17: 255-281, 1999. 